\title{
Schedule Adaptation for Ensuring Reliability in RT-WiFi-Based Networked Embedded Systems
}

\author{
YI-HUNG WEI, QUAN LENG, WEI-JU CHEN, and ALOYSIUS K. MOK, The University of \\ Texas at Austin, USA
}

SONG HAN, University of Connecticut, USA

\begin{abstract}
With the ever-growing interests in applying wireless technologies for networked embedded systems to serve as the communication fabric, many real-time wireless technologies have been recently developed to support time-critical sensing and control applications. We proposed in previous work the RT-WiFi protocol that provides real-time high-speed predictable data delivery and enables designs to meet time-critical industrial needs. However, without explicit reliability enforcement mechanisms, our previous RT-WiFi design is either subject to uncontrolled packet loss due to noise and other interferences or may suffer from inefficient communication channel usage. In this article, we explicitly consider interference from both Wi-Fi and non-Wi-Fi based interference sources and propose two sets of effective solutions for reliable data transmissions in RT-WiFi-based networked embedded systems. To improve reliability against general non-Wi-Fi based interference, based on rate adaptation and retransmission techniques, we present an optimal real-time rate adaption algorithm together with a communication link scheduler that has low network management overhead. A novel technique called overbooking is introduced to further improve the schedulability of the communication link scheduler while maintaining the required communication reliability. For Wi-Fi-based interference, we present mechanisms that utilize virtual carrier sensing to provide reliable data transmission while co-existing with regular Wi-Fi networks. We have implemented the proposed algorithms in the RT-WiFi network management framework and demonstrated the system performance with a series of experiments.
\end{abstract}

CCS Concepts: • Computer systems organization $\rightarrow$ Embedded systems; Redundancy; $\bullet$ Networks $\rightarrow$ Network reliability;

Additional Key Words and Phrases: Real-time systems, wireless networks, media access control

\section{ACM Reference format:}

Yi-Hung Wei, Quan Leng, Wei-Ju Chen, Aloysius K. Mok, and Song Han. 2018. Schedule Adaptation for Ensuring Reliability in RT-WiFi-Based Networked Embedded Systems. ACM Trans. Embed. Comput. Syst. 17, 5, Article 85 (October 2018), 23 pages.

https://doi.org/10.1145/3236011

\section{INTRODUCTION}

Networked embedded systems have drawn immense attention in recent years $[14,22]$ and have been widely deployed in a wide range of industrial sectors, including industrial automation [25],

Authors' addresses: Y.-H. Wei, Q. Leng, W.-J. Chen, and A. K. Mok, The University of Texas at Austin, Department of Computer Sciences, Austin, TX, 78712; emails: \{yhwei, qleng, albertwj, mok\}@cs.utexas.edu; S. Han, University of Connecticut, Computer Science and Engineering Department, Storrs, CT, 06269; email: song.han@uconn.edu.

Permission to make digital or hard copies of all or part of this work for personal or classroom use is granted without fee provided that copies are not made or distributed for profit or commercial advantage and that copies bear this notice and the full citation on the first page. Copyrights for components of this work owned by others than ACM must be honored. Abstracting with credit is permitted. To copy otherwise, or republish, to post on servers or to redistribute to lists, requires prior specific permission and/or a fee. Request permissions from permissions@acm.org.

(C) 2018 Association for Computing Machinery.

1539-9087/2018/10-ART85 \$15.00

https://doi.org/10.1145/3236011 
healthcare and biomedical systems [28], smart civil infrastructures [16], and intelligent robotic systems [7], to name a few. Compared to their wired counterparts, wireless networked embedded systems have been playing a more important role for exchanging critical monitoring and control information. This is mainly due to their great advantages of enhanced mobility, easier deployment as well as reduced maintenance and configuration costs.

Motivated by the varied communication requirements of wireless networked embedded systems, we proposed RT-WiFi [26], a real-time data link layer protocol that aims to support a wide range of applications. The design goal of RT-WiFi protocol is to support both predictable real-time data delivery with high-sampling-rates and application-customizable data link layer configuration. RT-WiFi networks adopt a centralized management mechanism that utilizes the time division multiple access (TDMA) channel access method for coordinating the channel access among RT-WiFi devices. By maintaining tight timing synchronization on each device, an RT-WiFi network divides the channel access time into small time slots, and explicitly allocates channel access time slots for each device to achieve predictable data delivery. Since the physical layer of RT-WiFi provides sufficient bandwidth, RT-WiFi can support up to $6 \mathrm{kHz}$ sampling rate. Furthermore, to meet diverse communication requirements of various wireless control applications, we design RT-WiFi to be a configurable platform to provide great flexibility to a wide range of control applications. For example, control engineers can customize their own communication design parameters that include sampling rate, transmission jitter, and reliability measures.

Although RT-WiFi can support predictable data delivery, how to provide reliable communication in noisy and harsh environments with stringent timing requirement is far from a completely resolved issue. Our previously proposed reliable transmission mechanisms [26] make pessimistic assumptions on the interference traffic, and our prior link scheduler [15] does not consider the communication characteristics of different data links. This can lead to inefficient channel usage, and unsatisfied system performance. However, most existing literatures either provides boundedtime data delivery under simplified reliability constraints or support reliable communication with limited real-time performance guarantees.

In this article, by explicitly considering interference from Wi-Fi and non-Wi-Fi based interference sources, we propose algorithms that adapt the communication schedule dynamically to support reliable real-time data delivery and avoid packet loss due to the presence of other interfere sources. By exploiting available mechanisms in the IEEE 802.11 [11] standard, such as rate adaptation, retransmission, and virtual carrier sensing manipulation, we allow the application designer to tailor the wireless system to meet application-specific real-time constraints on the communication links while meeting application-specific reliability requirements; we show how to implement the proposed solutions in COTS hardware.

The contribution of this work is summarized as follows:

- We formalize the real-time reliable link scheduling problem, and propose a real-time rate adaptation algorithm to drive optimal retransmissions with minimal transmission time that meets the expected reliability requirement.

- We present an efficient runtime communication link scheduler to schedule communication tasks with low network management overhead. Moreover, a novel technique called overbooking is invented that can significantly improve the schedulability of the link scheduler.

- To co-exist with regular Wi-Fi networks, we propose a virtual carrier-sensing-based mechanism that not only protects real-time data delivery in RT-WiFi networks but also shares unused bandwidth with regular Wi-Fi.

- We collect real world packet delivery rates in different scenarios and use these data to emulate the proposed algorithms to validate the practicability of our design and algorithms. Also, we create a testbed to compare proposed different co-existence mechanisms. 
The remainder of this article is organized as follows: Section 2 summarizes the related work. We describe RT-WiFi network architecture and system model in Section 3. Section 4 formalizes the reliable link scheduling problem, and presents a three-stage solution. Section 5 propose reliable communication mechanisms that further consider the interference from regular Wi-Fi networks. We describe our system implementation in Section 6 and summarize the experimental results in Section 7. Finally, we conclude the article and discuss the future work in Section 8.

\section{RELATED WORK}

Real-time wireless communication has drawn a lot of attention over the past decade. There have been extensive works that aim to provide reliable data transmission in regular Wi-Fi networks. For example, rate adaptation is an effective technique that adjusts the data rate of a link and employs a more error-resilient coding scheme for bad channel condition. Several works $[13,17,24$, 27] have been proposed to utilize rate adaptation to enhance reliability in wireless networks. However, those work focused on improving the overall network throughput, but did not differentiate the application-specific real-time constraints on individual flows. A flow scheduling framework is proposed in References $[9,10]$ and is applied to enhance the quality of service in Wi-Fi networks. Their focus, however, is on achieving long-term fairness of network flows, instead of enhancing real-time, short-horizon transmission reliability.

Several works $[2,3,8,23]$ have been proposed to provide reliable transmissions in both IEEE 802.11-based and 802.15.4-based real-time wireless network. Reference [23] proposes an IEEE 802.11 based TDMA data link layer protocol that reduces data collisions and adapts its transmission schedule to channel conditions. Reference [3] presents an admission control mechanism for IEEE 802.11 networks that considers the transmission errors and medium access delays caused by uncontrolled traffic. Reference [8] considers the reliable transmission in multi-hop real-time wireless mesh networks, and discusses how to construct reliable routing graphs and assigned communication schedules accordingly. Reference [2] considers the interference graph and proposes reliable communication schedulers for multi-hop networks. However, these literature studies focus on optimizing a specific reliability requirement. They do not consider the rate adaptation mechanism and interference from Wi-Fi networks.

There are several works in the non-preemptive scheduling literature that can be applied to the reliable data link scheduling problem. If the transmission time of each communication link is predetermined, then the techniques for scheduling non-preemptive tasks on a CPU can be applied to schedule communication links on TDMA-based wireless networks. Reference [12] shows that the problem is NP-hard in the strong sense to schedule a set of non-preemptive concrete task sets on a uni-processor. If the tasks are not strictly periodic, then Reference [1] shows that the nonpreemptive scheduling problem remains NP-hard even if the task periods are harmonic. Using the knowledge of incoming tasks, Reference [5] proposes a scheduler to insert idle time for increasing schedulability. Both References [1] and [21] present optimal schedulers with certain strong restrictions on the task periods. However, the periods of communication links usually do not meet their assumptions. If the tasks are strictly periodic, then Reference [19] provides sufficient conditions for scheduling task sets with both harmonic and non-harmonic periods. Reference [4] presents heuristics to determine the minimal number of processors to schedule a harmonic task set. All the aforementioned literature studies provide great insights of the difficulty of the link scheduling problem. In addition to studying schedulability of communication links, this article considers the link interference from both $\mathrm{Wi}-\mathrm{Fi}$ and non-Wi-Fi interference sources and presents co-existence mechanisms between RT-WiFi and regular Wi-Fi networks and a real-time rate adaption to provide real-time and reliable communication. 


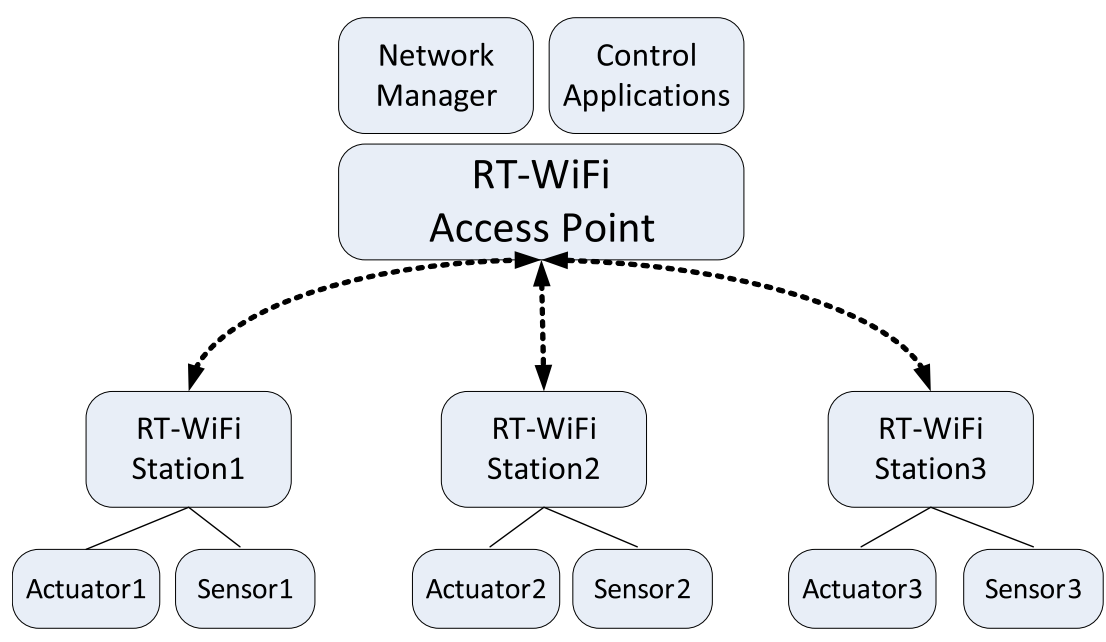

Fig. 1. An example of RT-WiFi-based networked control systems.

\section{RT-WIFI ARCHITECTURE AND SYSTEM MODEL}

\subsection{RT-WiFi Network Architecture}

RT-WiFi [26] is a real-time data link layer protocol that aims to support real-time high-speed wireless control systems with predictable packet delivery. Figure 1 shows a typical architecture of a wireless control system that adopts RT-WiFi as the communication infrastructure. An RT-WiFi network has the following three key components:

RT-WiFi Station: The RT-WiFi station is a device equipped with IEEE 802.11 compatible hardware and RT-WiFi protocol stack. In a RT-WiFi-based networked embedded system, the system designer connects sensors/actuators to the nearest RT-WiFi station for accessing the wireless network. To guarantee predictable real-time data delivery, the communication schedule of a station is configured by the network manager when it joins an RT-WiFi network.

RT-WiFi Access Point (AP): The RT-WiFi network manager resides in the application layer of the RT-WiFi AP, and all data and network management messages are exchanged through the RT-WiFi AP. The AP broadcasts timing and network management information in a beacon frame periodically.

Network Manager: The RT-WiFi network manager [15] is designed to coordinate allocation of shared network resource, manage the network dynamics, and construct the TDMA-based communication schedule. Depending on the network condition, the network manager dynamically updates the communication schedule and distributes the schedule to all the nodes in the RT-WiFi network.

\subsection{System Model}

In this article, we consider the basic single-cluster RT-WiFi network that consists of one RT-WiFi AP and multiple RT-WiFi stations as illustrated in Figure 1. The RT-WiFi AP and stations form a star network topology. Extension to more complex network topologies will be considered in a future article. The RT-WiFi network is a time-slotted system, and the basic time unit is a mini time slot, which is the minimal uninterruptible time unit in the system. The basic transmission unit in an RT-WiFi communication schedule is a time slot. A time slot consists of multiple mini time slots, and the size of a time slot is configurable based on the application requirements and channel condition. A communication link describes the communication behavior on a time slot, 
$\operatorname{Link1} \quad \operatorname{Link2} \quad \operatorname{Link3}$

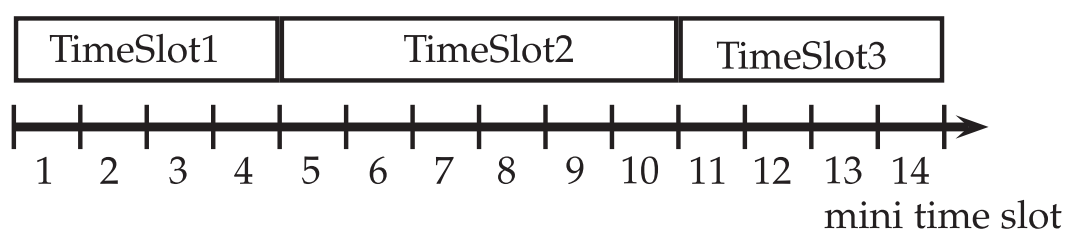

Fig. 2. An example of a superframe containing the schedule of three links of different time slot sizes. The schedule will repeat for every 14 mini time slots.

and it specifies the sender, receiver, and the timing information of the time slot. We classify links into two types. A downlink is a link with the AP as the sender, while an uplink is a link with the $\mathrm{AP}$ as the receiver. For example in Figure 2, TimeSlot1, TimeSlot2, and TimeSlot3 have a size of 4, 6, and 4 mini time slots and are associated with Link1, Link2, and Link3, respectively. Note that in RT-WiFi networks, different links may have time slots of different sizes.

The communication schedule of the RT-WiFi network is maintained by the network manager and is distributed by the AP periodically. The communication schedule is represented by a data structure called the superframe, which is a finite sequence of links that can be repeated infinitely to yield an infinite schedule at runtime. To adapt to network dynamics, the network manager updates the superframe to provide reliable communication. For example, in Figure 2, the superframe contains a schedule of 3 links and the schedule will repeat for every 14 mini time slots. For every beacon period, the AP compiles the communication schedule with other network management information (i.e., timing information) into a beacon frame and broadcasts it to the RT-WiFi network. Depending on the channel condition, the AP broadcasts the beacon frame one or multiple times in each period to ensure reliable delivery.

In this article, we abstract the wireless communication requirements using a set of communication links $L=\left\{L_{1}, L_{2}, \ldots, L_{n}\right\}$, where each link $L_{i}$ represents a communication task that transmits sensing/control data periodically. A communication link $L_{i}$ is characterized by three parameters $\left(P_{i}, D_{i}, B_{i}\right) . P_{i}$ and $D_{i}$ are positive integers in the unit of mini time slot, and they denote the period and deadline of a communication link $L_{i}$, respectively. In this article, we assume $P_{i}=D_{i}$ for simplicity of presentation. For a real-time communication link, a packet is successfully delivered if it is received by its deadline. $B_{i}$ denotes the packet size in bytes on link $L_{i}$. In our model, we assume that the set containing all the periods of the links forms a harmonic chain.

Definition 3.1. A set $\mathcal{S}$ of positive integers is a harmonic chain if and only if $\forall x, y \in \mathcal{S},(x \mid y) \vee$ $(y \mid x) \cdot x \mid y$ denotes $x$ divides $y$, or $y$ is a multiple of $x$.

RT-WiFi is designed to fulfill industry's need and it is suitable for a group of closed systems. We make this assumption based on practical system design considerations. In a real-world wireless sensing and control system, the periods of the links would not be set to arbitrary values. Application often may allow a range of sampling periods such as 100,200 , and $400 \mu \mathrm{s}$, and the harmonic periods of the communication tasks can be efficiently selected by period selection algorithms as proposed in the literatures $[15,20]$.

We introduce the following notations to model the transmission properties with different data rates. We assume that a set of $K$ data rates are supported in RT-WiFi system as $R=\left\{R_{1}, R_{2}, \ldots, R_{K}\right\}$, where $R_{j}$ denotes the number of bytes that the $j$ th data rate can transmit per mini time slot. For each communication link $L_{i}$, we use a set of tuple $\left\{\left(p_{(i, j)}, c_{(i, j)}\right)\right\}_{j=1}^{K}$ to represent the transmission properties with the given data rates. $p_{(i, j)}$ is the data delivery success rate of $L_{i}$ with data rate $R_{j}$, and $c_{(i, j)}$ is defined as the number of mini time slots needed to transmit the data link layer frame of 
size $B_{i}$ with data rate $R_{j}$. To accurately model the transmission time of a packet, we introduce $O$ to denote the transmission overhead. For each transmission, the overhead is represented as number of mini time slots and it consists of the time of inter frame space and ACK time. We can derive the transmission time $c_{(i, j)}$ of link $L_{i}$ as $c_{(i, j)}=O+\left\lceil B_{i} / R_{j}\right\rceil$.

To support reliable communication, we use $Q_{i}$ to denote the expected packet delivery ratio for link $L_{i}$. To achieve the expected packet delivery ratio, we can schedule a retry chain that consists of a series of data retransmissions with different available data rates. A retry chain of $L_{i}$ with $m_{i}$ retries is denoted as $T_{i}=\left\{T_{(i, 1)}, T_{(i, 2)}, \ldots, T_{\left(i, m_{i}\right)}\right\}$, where $1 \leq T_{(i, l)} \leq K$ for $1 \leq l \leq m_{i} . T_{(i, l)}$ represents the index of the data rates. For example, a retry chain $T_{i}=\{2,3\}$ indicates that $L_{i}$ will first transmit with data rate $R_{2}$. If the first transmission fails, it then uses data rate $R_{3}$ to retransmit it again. We define the transmission time and delivery rate of a retry chain as follows.

Definition 3.2. Given a retry chain $T_{i}=\left\{T_{(i, l)}\right\}_{l=1}^{m_{i}}$, the transmission time of $T_{i}$ is defined as $X\left(T_{i}\right)=\sum_{l=1}^{m_{i}} c_{\left(i, T_{(i, l)}\right)}$.

Definition 3.3. Given a retry chain $T_{i}=\left\{T_{(i, l)}\right\}_{l=1}^{m_{i}}$, the expected data delivery rate of $T_{i}$ is defined as $E\left(T_{i}\right)=1-\prod_{l=1}^{m_{i}}\left(1-p_{\left(i, T_{(i, l)}\right)}\right)$.

\section{RELIABLE TRANSMISSION FOR GENERAL INTERFERENCE SOURCES}

In this section, we discuss how to ensure desired quality of service in real-time reliable data transmission in the presence of general interference sources in RT-WiFi networks. We leverage on rate adaptation and retransmission mechanisms to construct a reliable TDMA communication schedule to provide reliable data transmission on a lossy channel. We first formulate the real-time reliable link scheduling problem and then present its computational complexity. Due to the hardness of this problem, we will present a three-stage approach to tackle it. For the last part of this section, we will address the channel condition monitoring mechanism, which keeps track of the delivery rate of each data rate.

\subsection{Real-Time Reliable Link Scheduling Problem}

Definition 4.1. Real-Time Reliable Link Scheduling (RTRLS) Problem: Given a set of data rates $R=\left\{R_{j}\right\}_{j=1}^{K}$, a set of communication links $L=\left\{L_{i}\right\}_{i=1}^{n}$ with a set of harmonic periods $P=\left\{P_{i}\right\}_{i=1}^{n}$, expected packet delivery ratio $Q=\left\{Q_{i}\right\}_{i=1}^{n}$, and each link $L_{i}$ is associated with its transmission properties $\left\{\left(p_{(i, j)}, c_{(i, j)}\right)\right\}_{j=1}^{K}$. The RTRLS problem is to determine a retry chain $T_{i}$ and a phasing $\phi_{i}$ for each link $L_{i}$, such that (C-1) $X\left(T_{i}\right) \leq D_{i}$ and $E\left(T_{i}\right) \geq Q_{i}(\mathbf{C}-2)$ the $s$ th instance of link $L_{i}$ is transmitted non-preemptively in $\left[\phi_{i}+s \cdot P_{i}, \phi_{i}+s \cdot P_{i}+X\left(T_{i}\right)\right)$.

In the RTRLS problem, we require the TDMA schedule of the communication links to be strictly periodic because of practical system implementation considerations. A strictly periodic schedule reduces the overhead of schedule distribution, simplifies interrupt handler design, and saves energy. We evaluate the schedule distribution overhead under different schedulers in Section 7.3.

THEOREM 4.1. To decide if an instance of the RTRLS problem is schedulable or not is NP-hard.

Proof. To show that the RTRLS problem is NP-hard, we reduce the 3-partition problem [6] into the RTRLS problem. As shown in Reference [6], the 3-partition problem is NP-Complete in the strong sense. The input of 3-partition problem is as following. Given a finite set $A$ of $3 m$ elements, a size $s(a) \in \mathbb{Z}^{+}$for each $a \in A$, a bound $B \in \mathbb{Z}^{+}$. Each $s(a)$ satisfies $\frac{B}{4}<s(a)<\frac{B}{2}$, and $\sum_{a \in A} s(a)=$ $m B$. The 3-partition problem asks if $A$ can be partitioned into $m$ disjoint sets $S_{1}, S_{2}, \ldots, S_{m}$ such that $\sum_{a \in S_{i}} s(a)=B$, where $1 \leq i \leq m$. 
We reduce an instance of the 3-partition problem into the RTRLS problem as follows. We create one data rate in the data rate set $R=\{1\}$. We construct link $L_{1}$ where $P_{1}=(B+1)$, and transmission condition $p_{(1,1)}=1.0$ and $c_{(1,1)}=1$. For each element $a \in A$, we create a communication link $L_{i}$ where $2 \leq i \leq 3 m+1, P_{i}=m(B+1)$, and transmission condition $p_{(i, 1)}=1.0$ and $c_{(i, 1)}=s(a)$. Under this setting, the periods of the communication links form a harmonic chain. For each element in $A$ it takes $O(1)$ time for the reduction. Thus, this reduction can be done in polynomial time.

If there exists a solution in 3-partition problem, then we set all the retry chains as $\left\{T_{i}=\right.$ $\{1\}\}_{i=1}^{3 m+1}$, and $\phi_{1}=0$. Suppose we order the elements in the disjoint set $S_{i}$ arbitrarily such that $S_{i}=\left\{s(a)_{(i, 1)}, s(a)_{(i, 2)}, \ldots, s(a)_{\left(i,\left|S_{i}\right|\right)}\right\}$, and for the oth element in $S_{i}$, we set the corresponding phasing as follows:

$$
\left\{\begin{array}{ll}
(i-1) \cdot(B+1)+1 & o=1 \\
(i-1) \cdot(B+1)+1+\sum_{k=1}^{o-1} s(a)_{i, k} & o>1
\end{array} .\right.
$$

This is a valid solution for the RTRLS problem since the expected delivery rate is fulfilled, and each link is transmitted non-preemptively.

However, if there is a solution for the RTRLS problem, we can add the corresponding element of links $\left\{L_{i}\right\}_{i=2}^{3 m+1}$ to a disjoint set $S_{k}$ depending on $\phi_{i}$ as follows:

$$
k= \begin{cases}\left\lfloor\frac{\phi_{i}-\phi_{1}}{(B+1)}\right\rfloor & , 1 \leq\left\lfloor\frac{\phi_{i}-\phi_{1}}{(B+1)}\right\rfloor \leq m \\ m & , \text { otherwise }\end{cases}
$$

It is a valid solution for the 3-partition problem, because $L_{1}$ is a strictly periodic task, and there are exactly $m$ mini time slots between two consecutive instance of $L_{1}$. Therefore, there is also a solution for the 3-partition problem. This completes the proof.

Since the RTRLS problem is NP-hard, we are more interested in finding a practical solution for our typical use case. We propose the following three-stage approach to tackle the RTRLS problem. In Section 4.2, we consider constraint C-1 in the RTRLS problem and present the real-time rate adaptation algorithm that generates a retry chain for each link to fulfill the expected reliability requirement with minimal transmission time. Given the transmission time of each link, we then consider constraint C-2 of the RTRLS problem and present a heuristic to construct the TDMA communication link schedule in Section 4.3. Finally, we introduce an overbooking technique in Section 4.4 to further improve the schedulability of the proposed scheduler.

\subsection{Stage 1: Real-Time Rate Adaptation}

We first present the real-time rate adaptation algorithm. The goal of this algorithm design is to derive a retry chain for a communication link such that the expected packet delivery rate is fulfilled and the transmission time of the retry chain is minimized. We formalize the rate adaptation problem as follows:

Definition 4.2. Real-Time Rate Adaptation (RTRA) Problem: Given $K$ data rates, and a communication link $L_{i}$ with deadline $D_{i}$, expected packet delivery ratio $Q_{i}$ and transmission properties $\left\{\left(p_{(i, j)}, c_{(i, j)}\right)\right\}_{j=1}^{K}$. The RTRA problem is to determine a retry chain $T_{i}=\left\{T_{(i, l)}\right\}_{l=1}^{m_{i}}$ such that $E\left(T_{i}\right) \geq Q_{i}, X\left(T_{i}\right) \leq D_{i}$ and $X\left(T_{i}\right)$ is minimized.

LEMMA 4.1. Given a time budget $t \leq D_{i}$, and we define $O P T_{i}(t)$ as the minimal packet loss rate for $L_{i}$. The OPT $(t)$ is determined by the following equation:

$$
\operatorname{OPT}_{i}(t)=\left\{\begin{array}{ll}
1.0 & \text { ift }=0 \\
\min \left\{\operatorname { m i n } _ { 1 \leq j \leq K } \left\{\operatorname{OPT}_{i}(t-1)\right.\right. & \\
\text { otherwise }
\end{array} .\right.
$$




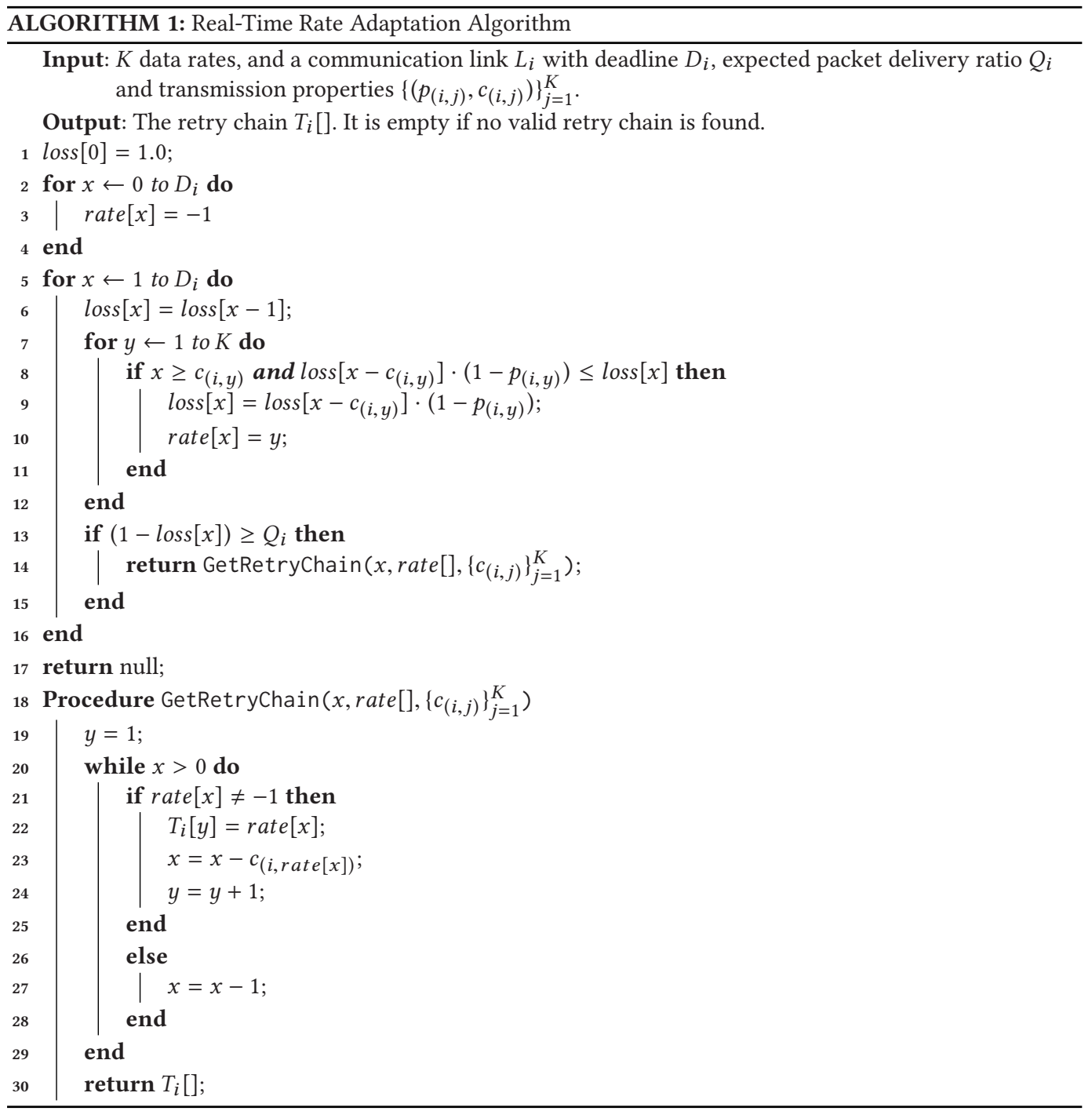

Proof. We prove this lemma by induction. For $t=0$, it is clear that the packet loss rate is 1.0 . Assuming that for $t=n$, the packet loss rate determined by Equation (1) is minimal. Then for $\operatorname{OPT}_{i}(n+1)$, there are two cases. For the first case, we simply use the same retry chain as $t=n$. Therefore, the loss rate is same as $O P T_{i}(n)$. For the second case, if the current time budget $(n+1)$ is greater or equal to the transmission time of data rate $R_{j}$, then we schedule $R_{j}$ at the end of the retry chain. The packet is lost if all the retries with time budget $t=(n+1)-c_{(i, j)}$ and the last retry failed. Thus, the minimal packet loss rate with $R_{j}$ is $O P T_{i}\left(n+1-c_{(i, j)}\right) \cdot\left(1-p_{(i, j)}\right)$. Then we select the minimal loss rate from all the $K$ data rates. Finally, we choose the smaller loss rate among the previous two cases as $\operatorname{OPT}_{i}(n+1)$, which is minimal. By mathematical induction the statement of Equation (1) holds for $1 \leq t \leq D_{i}$.

Based on the recursive substructure in Lemma 4.1, we propose a dynamic programming based algorithm that derives a retry chain with the minimal time budget. Algorithm 1 shows our 


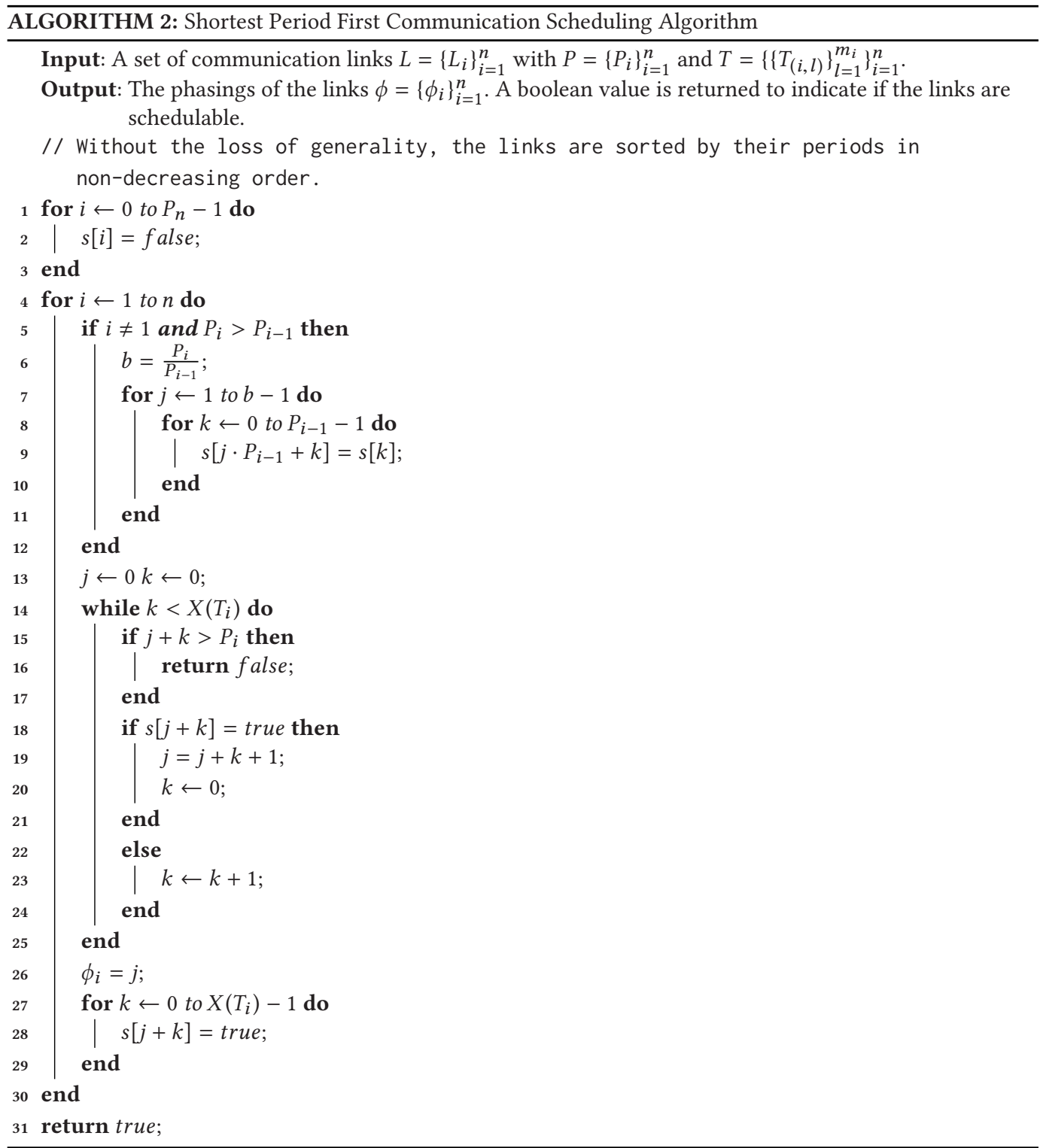

real-time rate adaptation (RTRA) algorithm. In this algorithm, array loss[] denotes the minimal packet loss rate under a particular time budget, and the array rates[] is used to derive a chain of data rates to achieve the minimal packet loss rate. We first initialize all arrays from line 1 to 4 . Line 5 to 16 are used to derive the minimal packet loss rate for time budget from 1 to $D_{i}$. As in line 6, the minimal packet loss rate for a time budget $x$ is at least as small as the loss rate with time budget $x-1$. In line 7 to 12 , we try all the $K$ data rates to test if we can achieve a lower packet loss rate. The loop would stop if the expected packet delivery rate is fulfilled in line 13 and we retrieve the retry chain in array $T_{i}$ by using procedure GetRetryChain(). If the expected packet delivery rate can not be met, then the algorithm will return null. The real-time rate adaptation algorithm is a pseudo-polynomial time algorithm. This is because for each time budget it takes $O(K)$ time 
to calculate the packet loss ratio of a data rate. Thus, time complexity of the RTRA algorithm is $O(D \cdot K)$.

\subsection{Stage 2: Communication Link Scheduling}

We now discuss the second stage for solving the RTRLS problem, that is how to schedule a set of communication links when their retry chains are determined. We focus on assigning phasings to the links such that condition C-2 in the RTRLS problem are fulfilled. We formally define the communication link scheduling problem as below.

Definition 4.3. Communication Link Scheduling (CLS) problem: Given a set of communication links $L=\left\{L_{i}\right\}_{i=1}^{n}$ with a set of harmonic periods $P=\left\{P_{i}\right\}_{i=1}^{n}$ and a set of retry chains $T=$ $\left\{\left\{T_{(i, l)}\right\}_{l=1}^{m_{i}}\right\}_{i=1}^{n}$. The CLS problem is to determine a set of phasings $\phi=\left\{\phi_{i}\right\}_{i=1}^{n}$ such that the $s$-th instance of link $L_{i}$ is transmitted non-preemptively during the interval $\left[\phi_{i}+s \cdot P_{i}, \phi_{i}+s \cdot P_{i}+\right.$ $\left.X\left(T_{i}\right)\right)$.

\section{THEOREM 4.2. To decide if an instance of the CLS problem is schedulable or not is NP-hard.}

Proof. It can be proved by reducing the 3-partition problem into the CLS problem and the proof is similar as the proof for Theorem 4.1.

As we discussed in Section 2, the CLS problem is similar to the problem of scheduling a set of non-preemptive strictly periodic tasks on a uni-processor system. Due to the hardness of the CLS problem, we focus on providing an efficient runtime algorithm for link scheduling with low network management overhead. We present our approach in Algorithm 2.

Algorithm 2 presents the structure of the communication link scheduling algorithm. Since the periods of the links form a harmonic chain, the hyperperiod of the links is the largest period among all the links. From line 1 to 3, we initialize a Boolean array $s[]$ of size $P_{n}$ to indicate if a phasing is assigned. From line 4 to 30 , we assign phasings to links in the non-decreasing order of period size. Since the task periods are in a harmonic chain, the schedule of a smaller period repeats multiple times in larger periods. From line 5 to 12 , if we detect $P_{i}$ is larger than $P_{i-1}$, we repeat the schedule in $\left[0, P_{i-1}-1\right] \frac{P_{i}}{P_{i-1}}$ times before we schedule link $L_{i}$. From line 14 to 25 , we loop through $P_{i}$ and check if there are enough unallocated slots that could fit $L_{i}$. If there are enough empty slots that can fit the retry chain $T_{i}$, then we assign $\phi_{i}$ for $L_{i}$ and mark down the schedule in line 27 to 29 . The time complexity of Algorithm 2 is $O\left(n \cdot P_{n}\right)$.

\subsection{Stage 3: Schedulability Enhancement with Overbooking}

In this section, we present a technique called overbooking to further enhance the schedulability of the communication link scheduling. The overbooking technique is based on a key observation that the last retry in a retry chain has relatively lower chance to be executed. We demonstrate the benefit of overbooking scheduling with a motivational example as shown in Figure 3. There are two links $L_{1}$ and $L_{2}$ with the same parameter settings as following. $P_{1}=P_{2}=D_{1}=D_{2}=5$, $Q_{1}=Q_{2}=0.8, p_{(1,1)}=p_{(2,1)}=0.5$, and $c_{(1,1)}=c_{(2,1)}=1$. By the proposed RTRA algorithm, we get $T_{1}=T_{2}=\{1,1,1\}$, and $E\left(T_{1}\right)=E\left(T_{2}\right)=0.875 \geq Q_{1}=Q_{2}$. Since $X\left(T_{1}\right)=X\left(T_{2}\right)=3$ and both of their deadlines are 5, we cannot schedule both links in Figure 3(a). However, we observe that the probability for $L_{1}$ to use the last retry is only 0.25 , because $L_{1}$ only uses the third retry if both the first two retries fail. Therefore, if there is an efficient mechanism that could detect $L_{1}$ 's usage of its last retry, then as shown in Figure 3(b), we can overbook mini time slot 3 such that $T_{(2,1)}$ only transmits if $T_{(1,3)}$ does not use the channel in mini time slot 3. Moreover, we need to make sure both $L_{1}$ and $L_{2}$ still meet their reliability requirement with the overbooking mechanism. For $L_{1}$, since $T_{(1,3)}$ is 


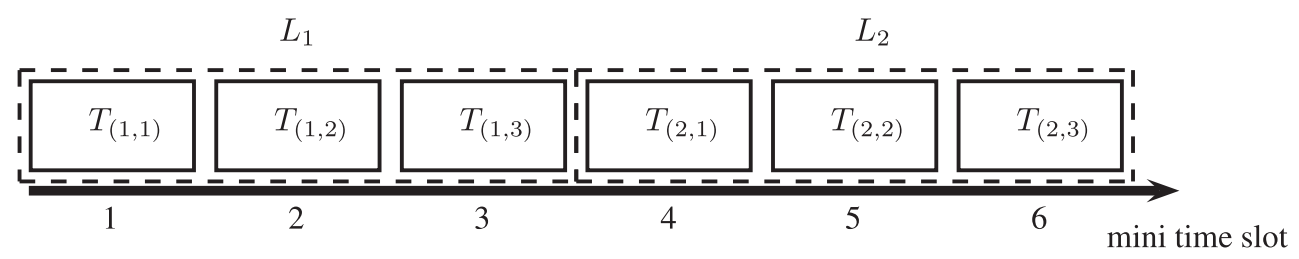

(a) Communication schedule without overbooking.

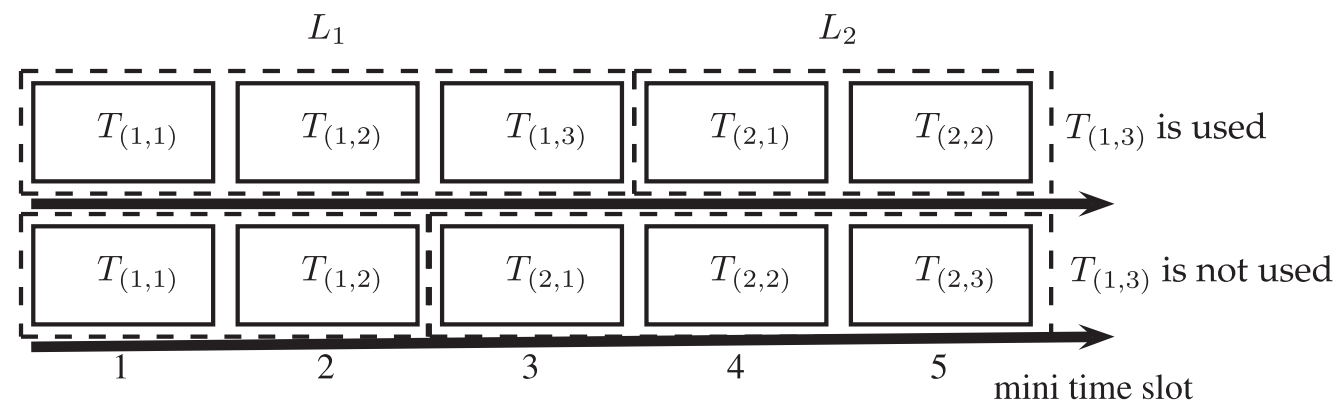

(b) Communication schedule with overbooking.

Fig. 3. Example of applying the overbooking mechanism.

always transmitted when it is necessary, the reliability requirement of $L_{1}$ is fulfilled. Additionally, since $L_{2}$ can only transmit at mini time slot 3 when $T_{(1,3)}$ is not transmitted, the successful packet delivery rate for $L_{2}$ is calculated as $0.75 \cdot E\left(\left\{T_{(2,1)}, T_{(2,2)}, T_{(2,3)}\right\}\right)+0.25 \cdot E\left(\left\{T_{(2,1)}, T_{(2,2)}\right\}\right)=$ $0.75 \cdot 0.875+0.25 \cdot 0.75=0.84375 \geq Q_{2}$. Thus, both $L_{1}$ and $L_{2}$ could be scheduled with expected reliability. In the following, we shall present the conflict resolution mechanism and elaborate how to construct a schedule with overbooking.

4.4.1 Efficient Conflict Resolution Mechanism with Overbooking. One way to reuse the spare time of an unused retry time is to let the network manager send a management message explicitly to notify the overbooked device to transmit. However, this message-based conflict resolution mechanism is inefficient, since the communication overhead of a notification message could be larger than the short unused spare time. Instead, we propose a novel approach that uses the characteristics of the wireless media to detect the unused spare time. We consider two links $L_{i}$ and $L_{j}$, assuming $L_{i}$ is already scheduled and we want to decide if $L_{j}$ can be scheduled right after $L_{i}$ with overbooking. Table 1 summarizes the conditions to resolve the conflict in the schedule with overbooking.

4.4.2 Overbooking Scheduling. Based on the aforementioned conditions, we can determine if a conflict in the overbooking schedule can be efficiently resolved without additional management overhead. We shall further calculate the transmission time and the expected packet delivery rate of a link $L_{j}$ if it is overbooked with its previous link $L_{i}$. In the following, we assume $L_{i}$ is already scheduled with $T_{i}=\left\{T_{(i, l)}\right\}_{l=1}^{m_{i}}$, and we want to schedule $L_{j}$ with the overbooking technique to satisfy $Q_{j}$.

We first introduce Algorithm 3 that can derive the retry chain $T_{i}$ with maximal packet delivery rate for a link $L_{i}$ with a given time budget $t_{i}$. This algorithm is modified from Algorithm 1, and it uses the GetRetryChain() procedure in Algorithm 1 to derive the retry chain. It has the same time complexity as Algorithm 1. We define Algorithm 3 as an operation, MaxRateRetry(), and we 
Table 1. A Summary of Overbooking Conditions

\begin{tabular}{|l|c|c|l|}
\hline$L_{i}$ & $L_{j}$ & Overboook & \multicolumn{1}{c|}{ Reason } \\
\hline Down link & Down link & Yes & $\begin{array}{l}\text { AP can only transmit } L_{j} \text { after it finishes } \\
\text { transmission of } L_{i} .\end{array}$ \\
\hline Down link & Up link & Yes & $\begin{array}{l}\text { Station that transmits } L_{j} \text { can carrier sense } \\
\text { AP's transmission of } L_{i} .\end{array}$ \\
\hline Up link & Down link & Yes & $\begin{array}{l}\text { AP can only transmit } L_{j} \text { after it finishes } \\
\text { receiving } L_{i} .\end{array}$ \\
\hline Up link & Up link & Depends & $\begin{array}{l}\text { Only if the source node of } L_{i} \text { and } L_{j} \text { are the } \\
\text { same station. Otherwise, the two stations } \\
\text { may not carrier sense each other. }\end{array}$ \\
\hline
\end{tabular}

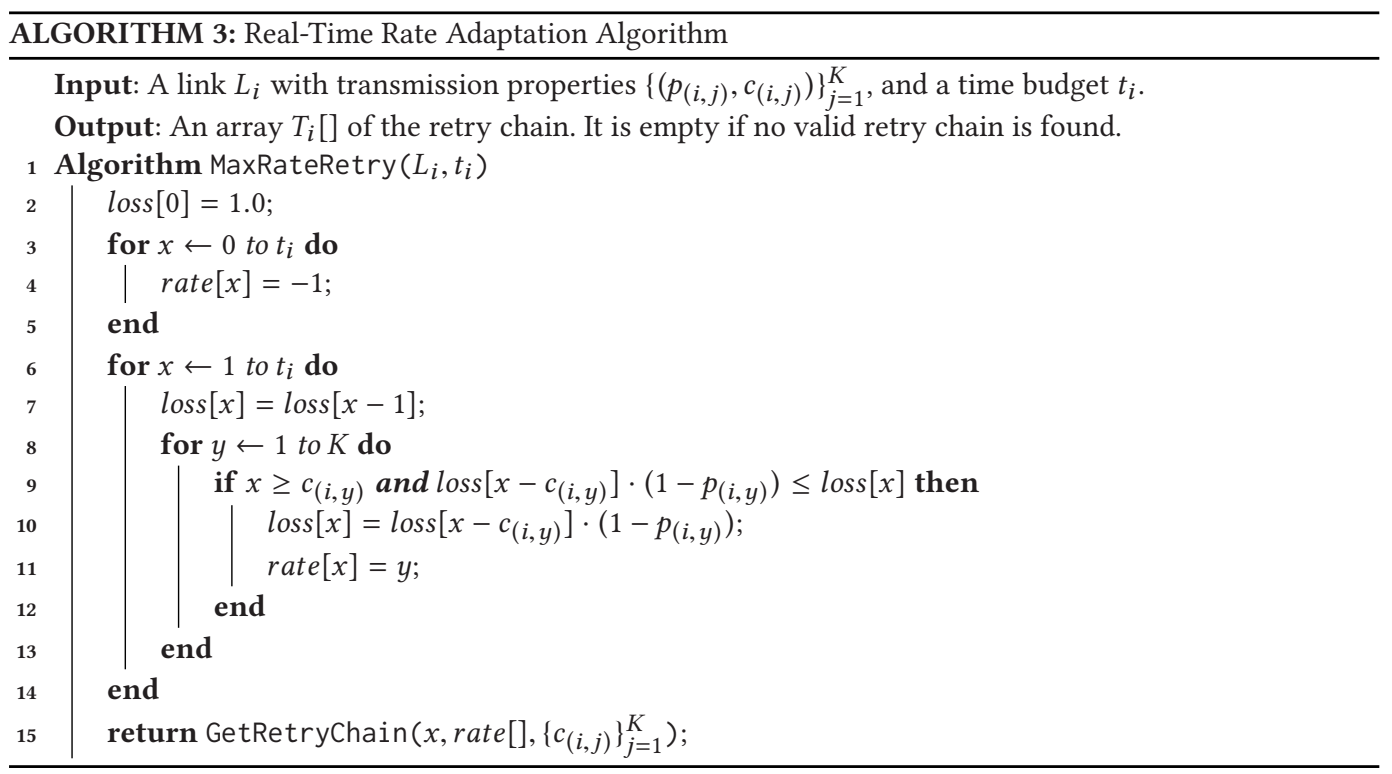

derive a retry chain as $T_{i}=\operatorname{MaxRateRetry}\left(L_{i}, t_{i}\right)$. We then define prob $b_{i}$ as the probability if $L_{i}$ does not access the channel in its last retry. $T_{j}$ and $T_{j}^{\prime}$ represent the retry chain of $L_{j}$ when $L_{i}$ does not access the channel in its last retry and when $L_{i}$ utilizes its last retry, respectively,

$$
\operatorname{prob}_{i}=1-\prod_{j=1}^{m_{i}-1}\left(1-p_{\left(i, T_{(i, j)}\right)}\right) .
$$

Depending on whether $L_{i}$ utilizes its last retry, we minimize the time budget $t_{j}$ as follows:

$$
\begin{array}{ll}
\text { minimize } & t_{j} \\
\text { subject to } & T_{j}=\operatorname{MaxRateRetry}\left(L_{j}, t_{j}+c_{\left(i, m_{i}\right)}\right) \\
& T_{j}^{\prime}=\operatorname{MaxRateRetry}\left(L_{j}, t_{j}\right) \\
& \operatorname{prob}_{i} \cdot E\left(T_{j}\right)+\left(1-\operatorname{prob}_{i}\right) \cdot E\left(T_{j}^{\prime}\right) \geq Q_{j} .
\end{array}
$$




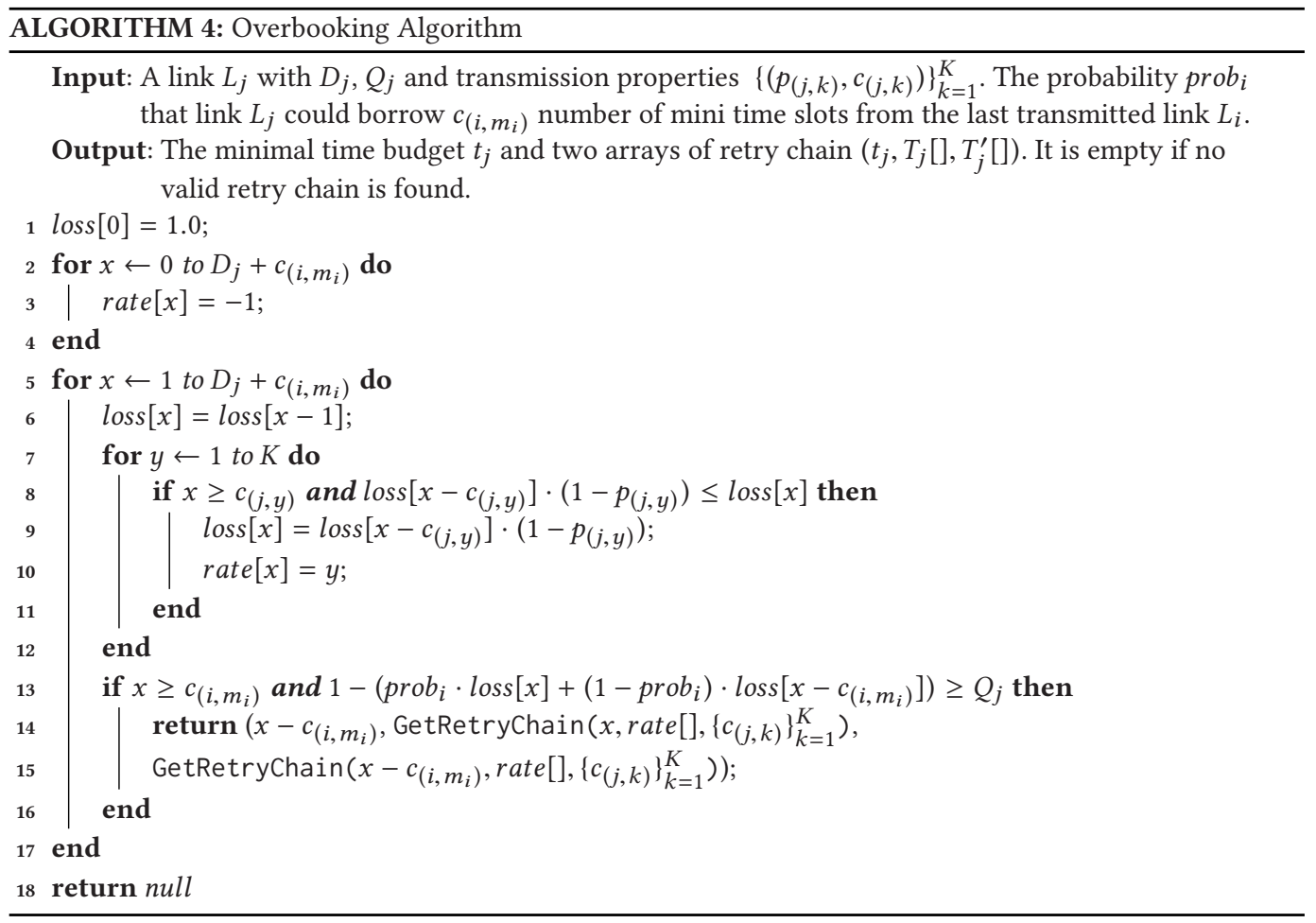

To calculate the minimal time budget $t_{j}$, we modify Algorithm 3 to perform checking along with computing the loss[], and stop when $Q_{j}$ is met as shown in Algorithm 4 from line 13 to 15. Therefore, the time complexity of the overbooking algorithm with minimal time budget remains the same as the RTRA algorithm. Please note that in the worst case, the overbooking technique falls back to the original RTRA algorithm when $\operatorname{prob}_{i} \rightarrow 0$. The overbooking technique effectively reuses the unused channel time and improves schedulability. We shall evaluate its performance in Section 7.3.

\subsection{Channel Condition Monitoring}

In this section, we address the channel condition monitor mechanism of the proposed real-time rate adaptation algorithm. The real-time rate adaptation algorithm takes $p_{(i, j)}$, the delivery rate of link $L_{i}$ with data rate $R_{j}$, as the input and these data delivery rates will be updated online in real time. For the regular $\mathrm{WiFi}$, the rate control algorithm will select $10 \%$ of the frames to probe the delivery rate for different data rates beside the optimal data rate used for transmission. The delivery rates of the recently transmitted frames will be monitored and the optimal data rate can be decided for the next frames to be transmitted. The real-time rate adaptation algorithm inherently selects different data rates for each scheduled link transmission to meet its requirement and the RT-WiFi will keep updating the delivery rate of each data rate. Also, RT-WiFi can actively probe the delivery rate of a link if that data rate has not been used recently to keep the delivery rate up to date. Based on these delivery rates updated during runtime, the real-time rate adaptation algorithm will compute a new schedule and broadcast it to all stations through the beacon frames for every $100 \mathrm{~ms}$. 


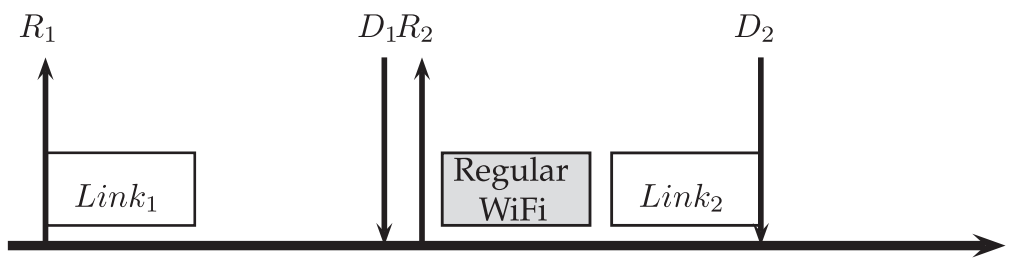

(a) Original co-existence mode in [26].

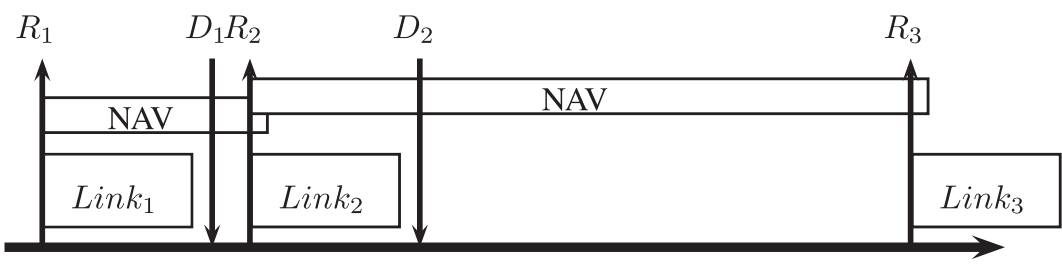

(b) Co-existence mode with virtual carrier sensing.

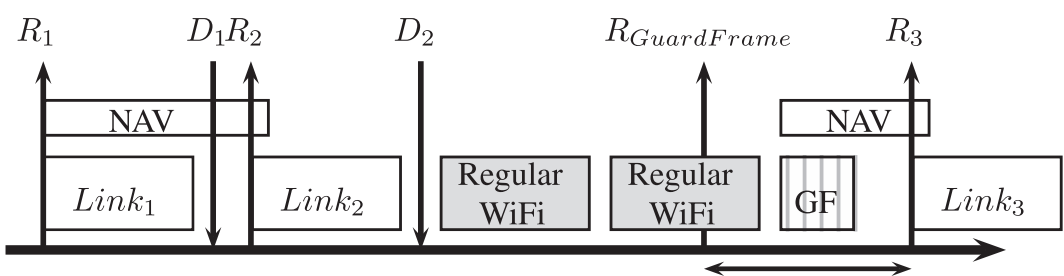

(c) Co-existence mode with bandwidth sharing.

Fig. 4. Three co-existence modes between RT-WiFi networks and regular Wi-Fi networks. $R_{i}$ and $D_{i}$ denote the release time and deadline of the $\operatorname{Link}_{i}$.

\section{CO-EXISTENCE WITH REGULAR WI-FI NETWORKS}

In this section, we discuss how to provide reliable data transmission for an RT-WiFi network when it co-exists with regular Wi-Fi networks. We assume the nodes in both RT-WiFi networks and regular Wi-Fi networks can carrier sense each other. Based on this assumption, we propose mechanisms to prioritize the data transmissions in RT-WiFi networks and share the remaining bandwidth with regular Wi-Fi networks.

\subsection{Deferring Wi-Fi Traffic with Virtual Carrier Sensing}

Our first proposed mechanism is to utilize virtual carrier sensing to actively defer the transmission of regular Wi-Fi traffic. In the IEEE 802.11 standard [11], virtual carrier sensing provides an indication of a busy media, and a regular Wi-Fi node defers its media access if it either physically or virtually senses that the media is busy. Virtual carrier sensing is implemented by the network allocation vector (NAV) mechanism. When a MAC layer frame is transmitted, it estimates the frame transmission time in micro seconds and specifies the time in the duration field in the MAC frame header. If a node receives a Wi-Fi MAC layer frame, then the node will set its NAV and keep the virtual carrier sensing on till the end of the duration. The maximum length of this duration can be set is $32,767 \mu$ s.

We compare the new virtual carrier sensing approach with the original co-existence mode that we proposed in Reference [26]. As shown in Figure 4(a), the old co-existence mode in Reference [26] cannot defer the transmission of regular Wi-Fi traffic, it pessimistically estimates the worst case 
transmission time of the regular Wi-Fi traffic assuming regular Wi-Fi uses the maximum transmission unit size and the lowest data rate. The original co-existence mode will need to have the deadlines of each link to take regular Wi-Fi transmission time into consideration and thus leads to inefficient channel usage. However, in Figure 4(b), we can use the NAV specified by Link $k_{i}$ to reserve the network channel such that regular Wi-Fi traffic will not be able to step in between the end of the $L_{i n k_{i}}$ transmission and the start of the $L_{i n k_{i+1}}$ transmission. In this way, we can schedule $L_{i n k_{i+1}}$ right after the deadline of $\operatorname{Link}_{i}$ even if $\operatorname{Link}_{i}$ does not use all of its retry chain, and regular Wi-Fi will be deferred till the end of $\operatorname{Link}_{i+1}$ transmission unless there is another NAV. For example, the NAV specified by Link $k_{1}$ will defer the data transmission of regular Wi-Fi network and this makes sure that the transmission of Link 2 will not be blocked by regular Wi-Fi network.

\subsection{Sharing Unused Bandwidth with Regular Wi-Fi Networks}

Although virtual carrier sensing can effectively defer the data transmission of regular Wi-Fi networks, aggressively using virtual carrier sensing may completely block traffic from regular Wi-Fi networks. For example, in Figure 4(b), to let the transmission of Link 3 not blocked by regular Wi-Fi traffic, NAV specified by Link $k_{2}$ will block the channel from the deadline $D_{2}$ of Link $k_{2}$ to the release time $R_{3}$ of $\operatorname{Link}_{3}$. This actually wastes the sharing opportunity for regular Wi-Fi traffic even if the RT-WiFi only uses a very small portion of the channel time. In the following, we present a mechanism to protect RT-WiFi traffic and share unused bandwidth with regular Wi-Fi networks.

Our bandwidth sharing mechanism consists of guard frame injection and the channel usage estimation. The guard frame is a management frame in the RT-WiFi network that broadcasts NAV to defer transmission of traffic from regular Wi-Fi networks. The channel usage estimation scheme logs the past channel access time of regular Wi-Fi traffic by measurement and it estimates the access time by using exponential weighted moving average. Suppose the estimated channel access time for regular Wi-Fi traffic is $t_{\text {Est }}$, and the transmission time of a guard frame is $t_{\text {GuardFrame, we }}$ define the sharing threshold $t_{\text {Shared }}$ as follows:

$$
t_{\text {Shared }}=t_{\text {Est }}+t_{\text {GuardFrame }} \text {. }
$$

As shown in Figure 4(c), if the channel idle time, which is from $D_{2}$ to $R_{3}$, is less than $t_{\text {Shared }}$, we use virtual carrier sensing to protect RT-WiFi traffic. However, if the channel idle time in our communication schedule exceeds the sharing threshold, we schedule a guard frame and share the used channel time to regular Wi-Fi traffic. Notice that because we reserve time to tolerate both the guard frame and the channel access from regular Wi-Fi traffic, the transmission of RT-WiFi traffic will not be delayed if the estimation is accurate. However, if the transmission of an RT-WiFi link misses its deadline because we underestimate the channel access time, then our system will update the channel access estimation adaptively and construct a new communication schedule.

\section{SYSTEM IMPLEMENTATION}

Figure 5 gives an overview of the software architecture of the RT-WiFi network management framework on both RT-WiFi AP and RT-WiFi station (STA). This design is built upon the Linux operating system and uses IEEE 802.11 compatible hardware. To meet tight timing constraints, we develop the RT-WiFi MAC module and RT-WiFi rate control module in the kernel space. The less time-critical components, such as the network manager and station agent are implemented in the user space to support easy porting and reconfiguration to other hardware platforms. To be backward compatible to existing system design, we integrate the RT-WiFi management framework with two existing kernel modules (mac80211 and hardware-dependent MAC) in the kernel space. Mac80211 is a kernel module that manages MAC sublayer management entity for IEEE 802.11 protocol in Linux, and the hardware dependent MAC kernel module is a device driver for 


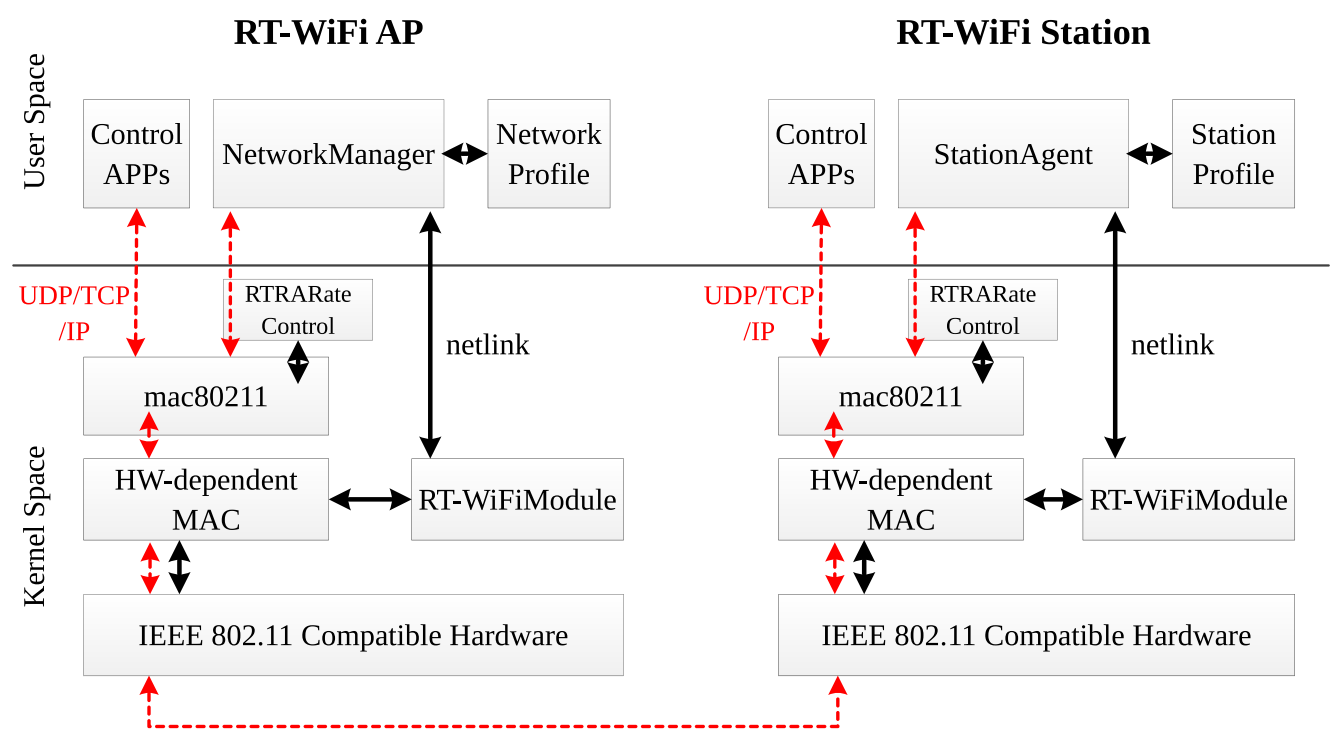

Fig. 5. An overview of the RT-WiFi software architecture.

handling a specific wireless network interface. Other than modifying the existing kernel module, we develop the RT-WiFi kernel module that is a configurable TDMA data link layer as presented in Reference [26]. The RT-WiFi kernel module is further enhanced with generic netlink socket to communicate with network manager and station agent in the user space.

There are two types of message flows in this network management architecture. The solid lines in Figure 5 represent the internal messages that are exchanged through internal interface within the RT-WiFi AP or RT-WiFi STA. The dashed lines show the flow of messages that are transmitted from one device to another. For the internal messages, netlink socket is used for communication between user space programs and kernel modules. Messages across different devices are transmitted with TCP or UDP protocols.

To support the proposed reliable transmission mechanisms, we implement the real-time rate adaptation algorithm using the rate control framework in mac80211. The communication link scheduler with the overbooking mechanism is developed in the network manager in the user space. The RT-WiFi kernel module logs packet delivery information, and updates the communication statistics to the network manager periodically. Depending on the network dynamics, the network manager builds a new communication schedule, and updates it to the RT-WiFi module in the AP. The AP then broadcasts the new schedule to the whole network in the next beacon period. Based on the sharing threshold, the network manager controls NAV of data frames, and inserts guard frames to the communication schedule accordingly. For ath9k wireless device driver, we manipulate the transmit descriptor to generate a guard frame.

\section{PERFORMANCE EVALUATION}

We have implemented the proposed algorithms, and conducted a series of experiments to evaluate the system performance. In this section, we first describe our experimental setup, present two sets of simulations and emulations to evaluate the performance of the real-time rate control algorithm and the communication link scheduler. We then present experiments on our testbed to evaluate the effectiveness of the co-existence mechanisms with regular Wi-Fi networks. 


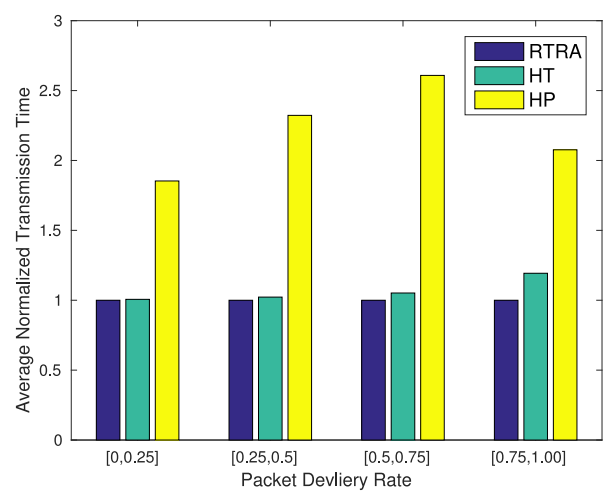

Fig. 6. Rate adaptation performance with different channel conditions.

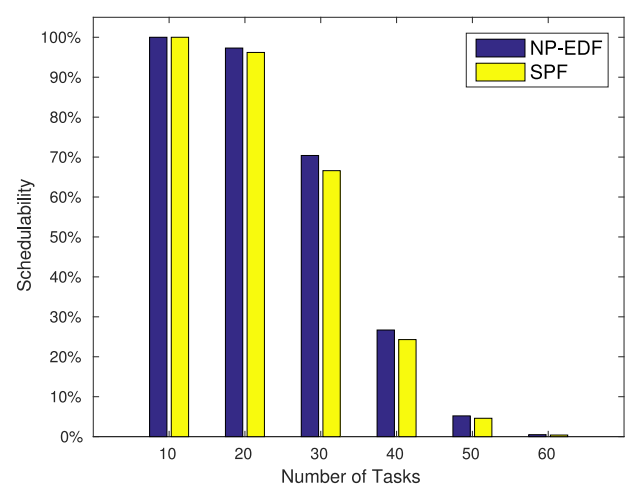

Fig. 7. Schedulability comparison: NP-EDF vs. SPF (trace NOON).

\subsection{System Setup}

The following system setup and parameter settings are used in our experiments. We run the simulations in Section 7.2 and Section 7.3 on a Linux desktop running Ubuntu 14.04 with 3.19 kernel and a Intel Core i7-2600 CPU, and we derive parameters from IEEE 802.11 standard [11] for our simulation. We use IEEE 802.11a as an example, and the parameters may be derived similarly for other physical layer technologies. There are 8 available data rates in IEEE 802.11a, ranging from $6 \mathrm{Mbps}$ to $54 \mathrm{Mbps}$. For each retry, the communication overhead $O$ consists of a PIFS, a SIFS, and an ACK timeout, which is $25+16+25=66(\mu \mathrm{s})$ in total. In the RT-WiFi protocol, the mini time slot size is set as $100 \mu \mathrm{s}$, and the expected packet delivery ratio for each link is 0.9 . For the experiments in Section 7.4, we run them on the testbed in our laboratory at University of Texas at Austin. We deploy one RT-WiFi AP on a Linux laptop with Ubuntu 14.04, 3.19 kernel and an Intel Core i3 2320M CPU, and we use six Intel Galileo boards as the RT-WiFi stations, which also have Ubuntu 14.04 and 3.19 kernel. Each RT-WiFi node is equipped with an Atheros AR9280 wireless card.

\subsection{Performance Evaluation on the Rate Adaptation Algorithm}

In this subsection, we evaluate the performance of the proposed real-time rate adaptation (RTRA) algorithm. In each simulation, we randomly generate 100,000 links to evaluate the performance of the rate adaptation algorithm under various channel conditions. We compare the RTRA algorithm with Linux kernel's default rate control algorithm minstrel [18]. While minstrel is not aware of deadline of a link, we utilize two heuristics that minstrel uses to construct retry chains and compare the transmission time of the retry chains constructed by RTRA and minstrel. The two heuristics, high throughput (HT) and high probability (HP), use the data rate with the highest throughput and highest packet delivery rate to build retry chains respectively. We construct retry chains to meet the expected packet delivery rate for the three methods, and compare the transmission time of the retry chains. Since the RTRA algorithm can always construct retry chains with minimum transmission time in the experiments, we report the transmission time of HT and HP heuristics normalized to the transmission time of RTRA.

In this set of experiments, we set the packet size to 1,500 bytes that is the maximum transmission unit (MTU) of the MAC layer, and compare the transmission time of retry chains with different packet delivery rates. We classify the ranges of packet delivery rates into four groups, which are $[0,0.25],[0.25,0.5],[0.5,0.75]$, and $[0.75,1.0]$. For each group, we generate communication links, and randomly assign the packet delivery rate for each data rate within the range of a group. We report the average normalized transmission time in Figure 6. As shown in Figure 6, RTRA and 
HT always outperform HP, because a data rate with high delivery rate does not always lead to short transmission time. In average, RTRA reduces packet transmission time over HT by around $20 \%$ when the packet delivery rate is within $[0.75,1.0]$, and they have similar average performance when the packet delivery rate is low. This is because the retry chain is longer with lower packet delivery rates, and the retry chain of RTRA is similar to HT in that case. However, in the worst case, we observe $10 \%$ to $55 \%$ transmission time reduction of RTRA over HT.

\subsection{Performance Evaluation on the SPF Link Scheduler}

We then evaluate the performance of our Shortest Period First (SPF) link scheduler based on real network condition traces. In the experiments, we focus on evaluating the schedulability and network management overhead of the proposed link scheduler. We compare SPF scheduler with NonPreemptive Earliest Deadline First (NP-EDF) scheduler [12]. For NP-EDF, we assume all links are ready at time 0 , and NP-EDF always schedules a link with the earliest deadline.

To emulate the network channel conditions monitored by the simulator, we collect the data delivery rates between one RT-WiFi AP and one RT-WiFi station. The captured traces record the delivery rate of each data rate for every $100 \mathrm{~ms}$ time interval. The traces will be randomly sampled to emulate the online channel conditions observed for the inputs of the proposed algorithm running for each beacon frame. We run the Linux tool perf to generate the traffic flow and record the delivery rates reported by the minstrel algorithm for every $100 \mathrm{~ms}$ time interval. We collected three traces at three different time of the same day that are 10:00-10:30 am, 14:00-14:30 and 18:00-18:30, respectively. We also denote the trace collected from 14:00-14:30 as trace NOON and we denote the aggregation of the three traces as trace ALL.

To evaluate the schedulability, we randomly generate 100,000 task sets and compare the number of schedulable task sets under these two algorithms. We vary the number of communication links to control the workload, and we select the parameters for communication links randomly according to the following uniform distributions. The packet size $B_{i} \in[100,1500]$ and the period $P_{i}$ in the following harmonic chain $=(2.5,5,10,50,100,200,1000) \mathrm{ms}$. For each packet transmission, the packet delivery rates $\left\{\left(p_{(i, j)}\right)\right\}_{j=1}^{K}$ are randomly selected from one of the data point in the trace. This harmonic chain represents sampling rates from $1 \mathrm{~Hz}$ to $400 \mathrm{~Hz}$, which are representative for various types of applications. To compare the network management overhead, we follow the network setup as described in Section 7.1. We assume it takes 6 bytes to specify a time slot in the TDMA schedule, which includes two bytes for the link ID, two bytes for time slot phasing, and two bytes for the time slot size.

Figures 7 and 8 report the percentage of schedulable task sets using SPF and NP-EDF schedulers using the trace NOON and ALL. When the number of tasks is between [10,20], both schedulers can schedule most of the task sets. NP-EDF outperforms SPF at most by 3 and $4 \%$, when there are 30 to 40 tasks in a task set for the trace NOON and ALL. When the number of tasks are more than 50 , because of the high workload, both schedulers can only schedule less than $10 \%$ of the total task sets under both settings. NP-EDF outperforms SPF, because a link does not require to be strictly periodic in NP-EDF schedule. Thus, a link can explore more phasings in NP-EDF scheduler than SPF scheduler. Figure 9 gives a comparison of the network overhead between the two approaches. We report the network overhead as the percentage of time to transmit the TDMA schedule frame in a beacon period. In Wi-Fi network, the default beacon period is $100 \mathrm{~ms}$. We transmit the beacon frame two times, to ensure that the management frame is reliably delivered. We observe the network management overhead for NP-EDF increases from $2 \%$ to $6 \%$ as the number of tasks increases. On the contrary, the network management overhead of SPF is significantly smaller than NP-EDF. SPF reduces the management overhead by $80 \%$ compared with NP-EDF, and it uses at most $1.2 \%$ channel time when the number of tasks is 60 . 


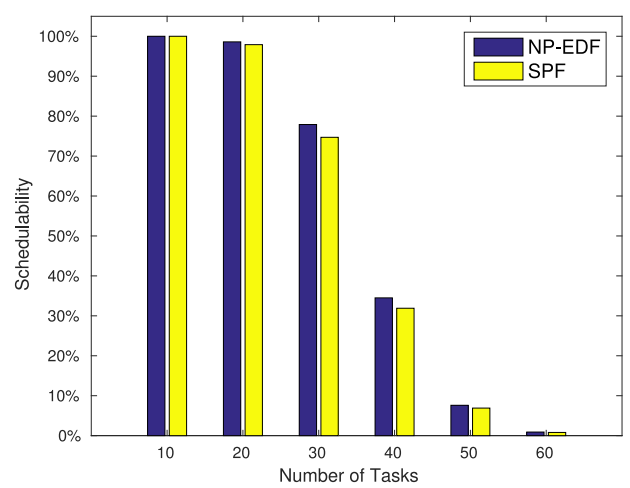

Fig. 8. Schedulability comparison: NP-EDF vs. SPF (trace ALL).

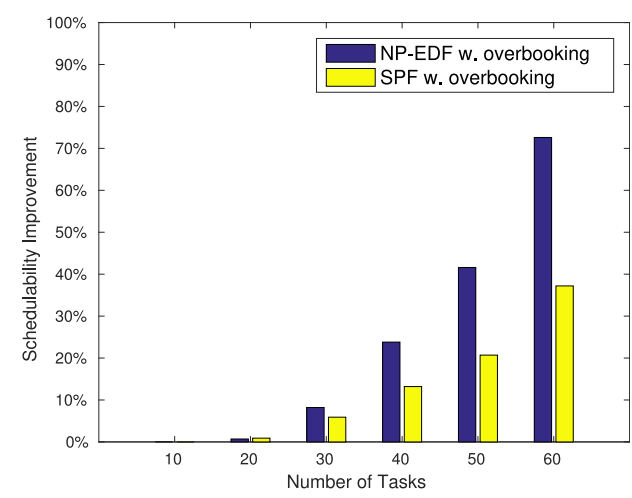

Fig. 10. Schedulability improvement w/ overbooking technique (trace NOON).

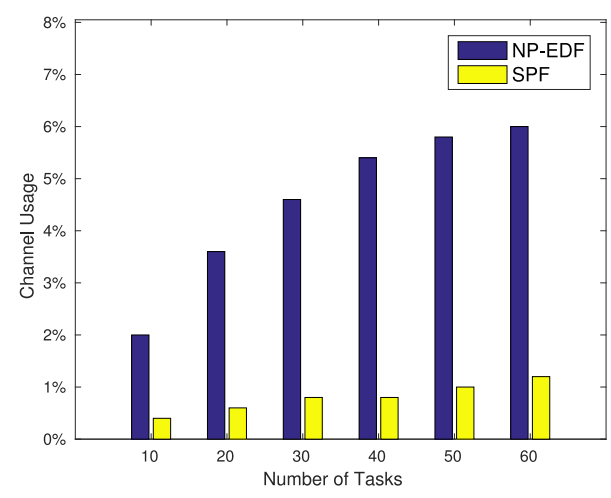

Fig. 9. Comparison of network management overhead.

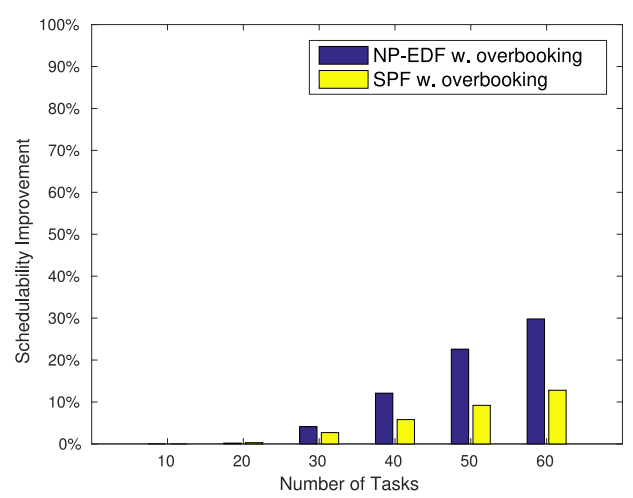

Fig. 11. Schedulability improvement w/ overbooking technique (trace ALL).

The main advantage of SPF is the network management overhead. Basically, SPF's network management overhead is strictly bonded by the number of tasks, while NP-EDF could be up to the size of the super periods of all the tasks. The super periods of the task set can be huge if the network bandwidth is large that it can accommodate more tasks. Arithmetically, SPF has clear advantage over NP-EDF but the difference in Figure 9 is not huge because of our available hardware and simulation emulation environment. We choose IEEE 802.11a as the physical layer in the simulation environment. In our experiment, the packet lost trace is based on the IEEE 802.11a physical layer, and we stick to this physical layer because of hardware limitation. The wireless card that we use is the commercially available hardware with open source driver that has the closest required features at that time. Since 802.11a is an old physical layer standard that can only support up to $54 \mathrm{Mbps}$. While the bandwidth of the latest Wi-Fi standard such as $802.11 \mathrm{ac}$ can be up to $866.7 \mathrm{Mbps}$, and 802.11ad claims to support more than $4 \mathrm{Gbps}$, we can image the performance gap between SPF and NP-EDF can be substantial.

We use the same task sets to evaluate the effectiveness of the overbooking mechanism. We define the schedulability improvement ratio (SIR) as follows:

$$
\operatorname{SIR}=\frac{N_{B}-N_{O}}{N_{O}} \cdot 100 \%
$$




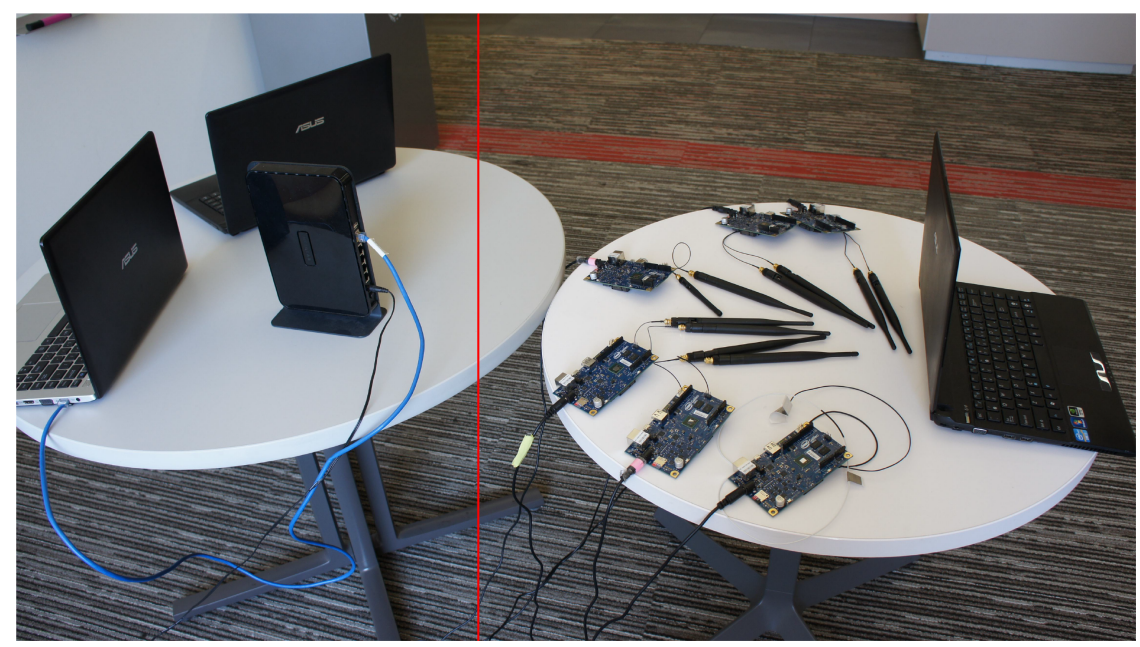

Fig. 12. Testbed with RT-WiFi networks on the right and regular WiFi networks on the left.

where $N_{B}$ and $N_{O}$ are the numbers of schedulable task sets with and without the overbooking technique, respectively. Figure 10 and 11 compare the SIR of NP-EDF and SPF schedulers with the overbooking technique. It can be clearly observed from the figures that when the number of tasks is small, the SIR is small, because the original scheduler can schedule most of the task sets already. The SIR increases along with the increase of the task numbers, because as the workload increases, a scheduler with overbooking technique can explore more scheduling opportunities. Also, comparing the improvement in Figure 10 and Figure 11, one can observe that when the network condition is worse (for example, using the trace NOON), the overbooking mechanism will have more significant schedulability improvement.

\subsection{Co-Existence Performance with Regular Wi-Fi Networks}

To test the effectiveness of the co-existence approach with the regular Wi-Fi network, we set up an RT-WiFi network and a regular Wi-Fi network as in Figure 12. The RT-WiFi network consists of one RT-WiFi AP and six RT-WiFi stations. We configure a NETGEAR WNDR3700 wireless router as a regular Wi-Fi AP that uses IEEE 802.11a on a $5 \mathrm{GHz}$ channel. To create a regular Wi-Fi link, one PC is connected to the router through Ethernet as the data receiver and another PC is connected to the router through regular Wi-Fi protocol as the data transmitter. Both the RT-WiFi and regular Wi-Fi networks are configured in an office environment using the same $5 \mathrm{GHz}$ channel. We only report the experiment results when the distance of the two networks is set to be $5 \mathrm{~m}$. Similar experiment result is observed when the distance between the two networks is within 10 meters. We acknowledge that if we increase the distance, various issues such as hidden terminal or multipath effect would degrade the effectiveness of carrier sense and the proposed scheme. However, these issues are more suitable to be resolved with physical layer solutions such as multiple input and multiple output (MIMO) and beam forming. The main focus of this article is the MAC layer scheduling. For the RT-WiFi network, we configure one link for each station. Because of limited computation power of the embedded Galileo board, we set the mini time slot to $256 \mu$ s. The period of each link is $40 \mathrm{mini}$ time slots, and we allocate 2 mini time slot transmission time for each link. The communication schedule is shown in Figure 13(a). For this schedule, we use iperf to generate the traffic for every link. On each RT-WiFi link, we generate a UDP flow with 1,450 bytes application layer payload at $1.133 \mathrm{Mbps}$ bit rate. To generate interference to the RT-WiFi network, we use iperf to configure 


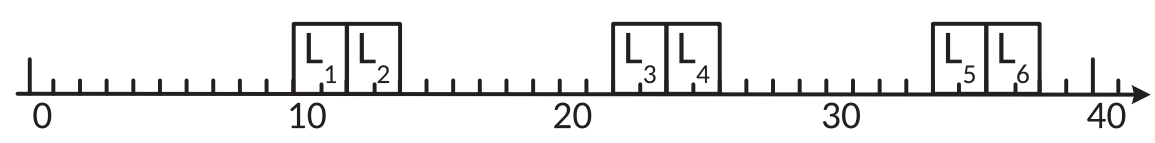

(a) A schedule without virtual carrier sensing.

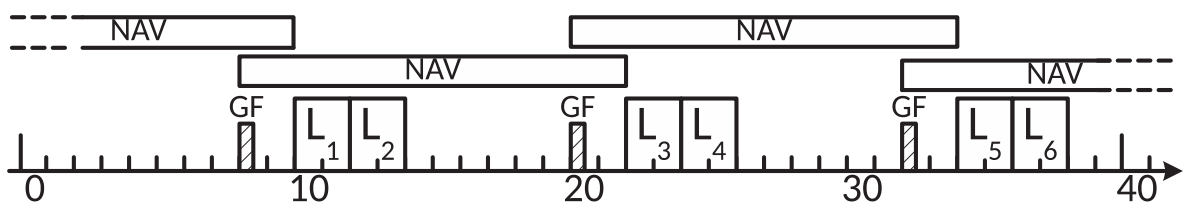

(b) A schedule with aggresive virtual carrier sensing.

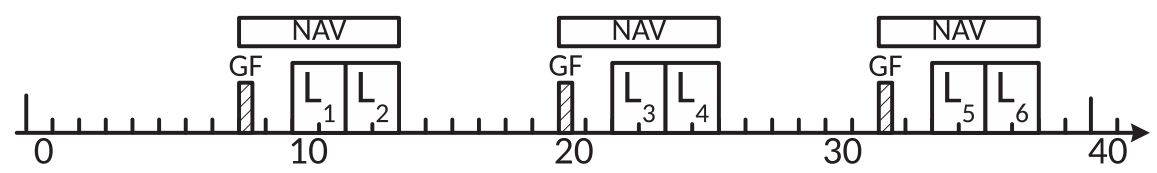

(c) A schedule with virtual carrier sensing and bandwidth sharing.

Fig. 13. Communication schedules of co-existence experiment.

Table 2. Comparison of Different Co-existence Mechanisms

\begin{tabular}{|c|c|c|c|c|c|c|c|}
\hline & \multicolumn{6}{|c|}{ Packet Loss Rate of RT-WiFi links } & $\begin{array}{c}\text { Regular Wi-Fi } \\
\text { link Throughput }\end{array}$ \\
\hline & $L_{1}$ & $L_{2}$ & $L_{3}$ & $L_{4}$ & $L_{5}$ & $L 6$ & $L_{\text {Regular Wi-Fi }}$ \\
\hline RT-WiFi baseline & $0.06 \%$ & $0.51 \%$ & $1.1 \%$ & $0.92 \%$ & $1.1 \%$ & $0.12 \%$ & N/A \\
\hline Regular Wi-Fi baseline & \multicolumn{6}{|c|}{ N/A } & $26.9 \mathrm{Mbps}$ \\
\hline $\begin{array}{l}\text { Both traffic } \\
\text { (No virtual carrier sensing) }\end{array}$ & $3.7 \%$ & $6.7 \%$ & $4.3 \%$ & $8.7 \%$ & $4.8 \%$ & $3.2 \%$ & $21.6 \mathrm{Mbps}$ \\
\hline $\begin{array}{l}\text { Both traffic } \\
\text { (With virtual carrier sensing) }\end{array}$ & $0.15 \%$ & $0.63 \%$ & $1.2 \%$ & $0.91 \%$ & $1.3 \%$ & $0.32 \%$ & Disconnected \\
\hline $\begin{array}{l}\text { Both traffic } \\
\text { (With virtual carrier sensing and } \\
\text { bandwidth sharing) }\end{array}$ & $0.75 \%$ & $0.77 \%$ & $1.8 \%$ & $1.0 \%$ & $1.7 \%$ & $0.32 \%$ & $13.4 \mathrm{Mbps}$ \\
\hline
\end{tabular}

another UDP flow with 1,450 bytes application payload at 30Mbps bit rate on the regular Wi-Fi link. For the following experiments, we run each experiment for 10 minutes. We report the packet loss rate of the RT-WiFi links and the throughput of the regular Wi-Fi link.

We summarize the co-existence experiment results in Table 2. The first two rows in Table 2 show the baseline measurement of the RT-WiFi links and the regular Wi-Fi link respectively. As shown in the third row, if the carrier sensing mechanism is disabled, we observe higher packet loss rates on all the RT-WiFi links. The fourth row shows the network performance when we use the virtual carrier sensing mechanism as shown in Figure 13(b). Since we allocate NAV aggressively in this schedule, the packet loss rates for RT-WiFi links are similar to those in the baseline. However, the regular Wi-Fi link is blocked completely. To share unused bandwidth with regular Wi-Fi network, we enable the bandwidth sharing mechanism as shown in Figure 13(c). With this communication schedule, we preserve similar packet delivery rates for RT-WiFi links, and let the regular Wi-Fi link retain around half of its baseline bandwidth. Note that, in this experiment the retransmission 
mechanism for RT-WiFi is disabled and there is packet loss due to environmental noise even when there is no regular Wi-Fi traffic.

\section{CONCLUSION AND FUTURE WORK}

Considering the interference from both Wi-Fi and non-Wi-Fi interference sources, this article proposes various solutions to support real-time reliable wireless communication for RT-WiFi networks. For general non-Wi-Fi interference, we apply rate adaptation and retransmission mechanisms to enhance the reliability mechanisms of the network manager that dynamically constructs and distributes communication schedules to the network. To build reliable communication schedules, we present an optimal rate control algorithm RTRA, a communication link scheduler SPF that has low network management overhead, and the overbooking technique that further improves the schedulability of the network link scheduler. For regular Wi-Fi based interference, we propose a virtual carrier sensing based approach that prioritizes transmission of RT-WiFi traffic and shares unused channel time with regular Wi-Fi network users. A series of simulations, emulations and testbed experiments are conducted to demonstrate the effectiveness of the proposed algorithms and mechanisms.

For future work, we will consider how to deploy RT-WiFi networks in a large area that requires more than one RT-WiFi cluster. The research issues include how to coordinate the inter-cluster interference across different RT-WiFi clusters, and how to support end-to-end performance guarantees across different RT-WiFi clusters.

\section{ACKNOWLEDGMENTS}

The work reported herein is partially supported by the Office of Naval Research under ONR Award N00014-17-2216 and by a gift from General Motors Corporation.

\section{REFERENCES}

[1] Yang Cai and M. C. Kong. 1996. Nonpreemptive scheduling of periodic tasks in uni- and multiprocessor systems. Algorithmica 15, 6 (1996), 572-599.

[2] O. Chipara, C. Wu, C. Lu, and W. Griswold. 2011. Interference-aware real-time flow scheduling for wireless sensor networks. In Proceedings of the Euromicro Conference on Real-Time Systems (ECRTS'11). 67-77.

[3] R. Costa, P. Portugal, R. Moraes, and F. Vasques. 2012. An admission control mechanism to handle real-time traffic in IEEE 802.11 networks in open communication environments. In Proceedings of the 2012 9th IEEE International Workshop on Factory Communication Systems (WFCS'12). 63-66.

[4] Friedrich Eisenbrand, Nicolai Hähnle, Martin Niemeier, Martin Skutella, José Verschae, and Andreas Wiese. 2010. Scheduling periodic tasks in a hard real-time environment. In Proceedings of the 37th International Colloquium on Automata, Languages and Programming (ICALP'10). 299-311.

[5] C. Ekelin. 2006. Clairvoyant non-preemptive EDF scheduling. In Proceedings of the 18th Euromicro Conference on Real-Time Systems (ECRTS’06). 7-32. DOI : http://dx.doi.org/10.1109/ECRTS.2006.7

[6] M. R. Garey and D. S. Johnson. 1979. Computers and Intractability: A Guide to the Theory of NP-Completeness. W. H. Freeman.

[7] S. Han, A. K. Mok, J. Meng, Y.-H. Wei, P.-C. Huang, Q. Leng, X. Zhu, L. Sentis, K. S. Kim, and R. Miikkulainen. 2013. Architecture of a cyberphysical avatar. In Proceedings of the International Conference on Cyber-Physical Systems (ICCPS'13).

[8] S. Han, X. Zhu, D. Chen, A. K. Mok, and M. Nixon. 2011. Reliable and real-time communication in industrial wireless mesh networks. In Proceedings of the IEEE Real-Time and Embedded Technology and Applications Symposium (RTAS'11). $3-12$.

[9] I. H. Hou, V. Borkar, and P. R. Kumar. 2009. A theory of QoS for wireless. In Proceedings of the IEEE Conference on Computer Communications (INFOCOM'09). 486-494.

[10] I. H. Hou and P. R. Kumar. 2010. Scheduling heterogeneous real-time traffic over fading wireless channels. In Proceedings of the IEEE Conference on Computer Communications (INFOCOM'10). 1-9.

[11] IEEE 802.11 working group. 2012. IEEE 802.11 Standard. Retrieved Feburary 26, 2017 from http://www.ieee802.org/ $11 /$. 
[12] K. Jeffay, D. F. Stanat, and C. U. Martel. 1991. On non-preemptive scheduling of period and sporadic tasks. In Proceedings of the IEEE Real-Time Systems Symposium (RTSS'91). 129-139.

[13] Glenn Judd, Xiaohui Wang, and Peter Steenkiste. 2008. Efficient channel-aware rate adaptation in dynamic environments. In Proceedings of the 6th International Conference on Mobile Systems, Applications, and Services (MobiSys'08). 118-131.

[14] Edward Lee and others. 2008. Cyber physical systems: Design challenges. In Proceedings of the 2008 11th IEEE International Symposium on Object Oriented Real-Time Distributed Computing (ISORC'08). IEEE, 363-369.

[15] Q. Leng, Y.-H. Wei, S. Han, A. K. Mok, W. Zhang, and M. Tomizuka. 2014. Improving control performance by minimizing jitter in RT-WiFi networks. In Proceedings of the IEEE Real-Time Systems Symposium (RTSS'14). 63-73.

[16] B. Li, Z. Sun, K. Mechitov, G. Hackmann, C. Lu, S. J. Dyke, G. Agha, and B. F. Spencer. 2013. Realistic case studies of wireless structural control. In Proceedings of the IEEE/ACM International Conference on Cyber-Physical Systems (ICCPS'13).

[17] C. Y. Li, C. Peng, S. Lu, X. Wang, and R. Chandra. 2015. Latency-aware rate adaptation in 802.11n home networks. In Proceedings of the IEEE Conference on Computer Communications (INFOCOM'15). 1293-1301. DOI : http://dx.doi.org/ 10.1109/INFOCOM.2015.7218505

[18] Linux wireless group. 2016. Minstrel. Retrieved Feburary 26, 2017 from https://wireless.wiki.kernel.org/en/ developers/documentation/mac80211/ratecontrol/minstrel.

[19] M. Marouf and Y. Sorel. 2011. Scheduling non-preemptive hard real-time tasks with strict periods. In Proceedings of the 2011 IEEE 16th Conference on Emerging Technologies \& Factory Automation (ETFA'11). 1-8.

[20] M. Nasri and G. Fohler. 2015. An efficient method for assigning harmonic periods to hard real-time tasks with period ranges. In Proceedings of the 2015 27th Euromicro Conference on Real-Time Systems. 149-159. DOI : http://dx.doi.org/10. 1109/ECRTS.2015.21

[21] Mitra Nasri and Mehdi Kargahi. 2014. Precautious-RM: A predictable non-preemptive scheduling algorithm for harmonic tasks. Real-Time Syst. 50, 4 (2014), 548-584. DOI : http://dx.doi.org/10.1007/s11241-014-9203-y

[22] Ragunathan (Raj) Rajkumar, Insup Lee, Lui Sha, and John Stankovic. 2010. Cyber-physical systems: The next computing revolution. In Proceedings of the 47th Design Automation Conference (DAC'10). ACM, New York, NY, 731-736.

[23] F. Santos, L. Almeida, P. Pedreiras, L. S. Lopes, and T. Facchinetti. 2004. An adaptive tdma protocol for soft realtime wireless communication among mobile autonomous agents. In Proceedings of the International Workshop on Architecture for Cooperative Embedded Real-Time Systems (WACERTS'04), Vol. 2004. 657-665.

[24] Souvik Sen, Naveen Santhapuri, Romit Roy Choudhury, and Srihari Nelakuditi. 2010. AccuRate: Constellation based rate estimation in wireless networks. In Proceedings of the 7th USENIX Conference on Networked Systems Design and Implementation (NSDI'10). 12-12.

[25] J. Song, S. Han, A. K. Mok, D. Chen, M. Lucas, M. Nixon, and W. Pratt. 2008. WirelessHART: Applying wireless technology in real-time industrial process control. In Proceedings of the IEEE Real-Time and Embedded Technology and Applications Symposium (RTAS'08). 377-386.

[26] Y.-H. Wei, Q. Leng, S. Han, A. K. Mok, W. Zhang, and M. Tomizuka. 2013. RT-WiFi: Real-time high-speed communication protocol for wireless cyber-physical control applications. In Proceedings of the IEEE Real-Time Systems Symposium (RTSS'13). 140-149.

[27] Starsky H. Y. Wong, Hao Yang, Songwu Lu, and Vaduvur Bharghavan. 2006. Robust rate adaptation for 802.11 wireless networks. In Proceedings of the 12th Annual International Conference on Mobile Computing and Networking (MobiCom'06). 146-157.

[28] Wenlong Zhang, Xiuming Zhu, Song Han, Nancy Byl, Aloysius K. Mok, and Masayoshi Tomizuka. 2012. Design of a network-based mobile gait rehabilitation system. In Proceedings of the IEEE International Conference on Robotics and Biomimetics (ROBIO'12). 1773-1778.

Received October 2017; revised February 2018; accepted June 2018 